\title{
Risk factor paradox: No prognostic impact of arterial hypertension and smoking in patients with ventricular tachyarrhythmias
}

\author{
Kathrin Weidner ${ }^{1 *}$, Michael Behnes ${ }^{1 *}$, Jonas Rusnak ${ }^{1}$, Gabriel Taton ${ }^{1}$, Tobias Schupp ${ }^{1}$, \\ Linda Reiser ${ }^{1}$, Armin Bollow ${ }^{1}$, Thomas Reichelt ${ }^{1}$, Dominik Ellguth ${ }^{1}$, Niko Engelke ${ }^{1}$, \\ Philipp Kuche ${ }^{1}$, Jorge Hoppner ${ }^{2}$, Ibrahim El-Battrawy ${ }^{1}$, Siegfried Lang ${ }^{1}$, \\ Christoph A. Nienaber ${ }^{3}$, Kambis Mashayekhi ${ }^{4}$, Dennis Ferdinand ${ }^{5}$, \\ Christel Weiss ${ }^{5}$, Martin Borggrefe ${ }^{1}$, Ibrahim Akin ${ }^{1}$
}

${ }^{1}$ First Department of Medicine, University Medical Center Mannheim (UMM), Faculty of Medicine Mannheim, University of Heidelberg, European Center for AngioScience (ECAS), and German Center for Cardiovascular Research (DZHK) partner site Heidelberg/Mannheim, Mannheim, Germany ${ }^{2}$ Clinic for Diagnostic and Interventional Radiology Heidelberg, University of Heidelberg, Germany

${ }^{3}$ Royal Brompton and Harefield Hospitals, NHS, London, United Kingdom

${ }^{4}$ Department of Cardiology and Angiology II, University Heart Center Freiburg, Bad Krozingen, Germany ${ }^{5}$ Institute of Biomathematics and Medical Statistics, University Medical Center Mannheim (UMM),

Faculty of Medicine Mannheim, University of Heidelberg, Mannheim, Germany

\begin{abstract}
Background: Data regarding the outcome of patients with ventricular tachyarrhythmias related to arterial hypertension (AHT) and smoking is limited. The study sought to assess the prognostic impact of AHT and smoking on survival in patients presenting with ventricular tachyarrhythmias.

Methods: All consecutive patients surviving ventricular tachycardia (VT) and ventricular fibrillation (VF) upon admission to the University Medical Center Mannheim (UMM), Germany from 2002 to 2016 were included and stratified according to AHT and smoking by propensity score matching. The primary prognostic endpoint was all-cause mortality at 30 months.

Results: A total of 988 AHT-matched patients (494 each, with and without AHT) and a total of 872 smoking-matched patients (436 each, with and without smoking) were included. The rates of VT and VF were similar in both groups (VT: AHT 60\% vs. no AHT 60\%; smokers $61 \%$ vs. non-smokers 62\%; VF: AHT 35\% vs. no AHT 38\%; smokers 39\% vs. non-smokers 38\%). Neither AHT nor smoking were associated with the primary endpoint of long-term all-cause mortality at 30 months (long-term mortality rates: $A H T / n o$ AHT, $26 \%$ vs. $28 \%$; log-rank $p=0.525$; smoking/non-smoking, $22 \%$ vs. $25 \%$; log-rank $p=0.683$.

Conclusions: Paradoxically, neither AHT nor smoking were associated with differences of long-term all-cause mortality in patients presenting with ventricular tachyarrhythmias. (Cardiol J 2020; 27, 6: 715-725)
\end{abstract}

Key words: ventricular tachyarrhythmias, arterial hypertension, smoking, long-term all-cause mortality

Address for correspondence: PD Dr. med. Michael Behnes, First Department of Medicine, University Medical Center Mannheim (UMM), Theodor-Kutzer-Ufer 1-3, 68167 Mannheim, Germany, tel: +49 621383 6239, fax: +49 621 3832012 , e-mail: michael.behnes@umm.de

Received: 30.07.2018 Accepted: 7.11.2018

*Kathrin Weidner and Michael Behnes contributed equally to this study. 


\section{Introduction}

Sudden cardiac death (SCD) related to ventricular tachyarrhythmias accounts for $15-20 \%$ of all deaths in the Western world and remains associated with poor clinical outcomes [1]. Therefore, it is important to stratify high-risk patients presenting with ventricular tachyarrhythmias according to their individual risk for future outcomes.

Arterial hypertension (AHT) and nicotine, a major component of cigarettes inhaled during smoking, are common cardiovascular risk factors for coronary artery disease (CAD) and heart failure (HF) [2-4]. Patients suffering from CAD and $\mathrm{HF}$ are at risk of developing life-threatening ventricular tachyarrhythmias $[2,5]$. Ventricular tachyarrhythmias are also associated with left ventricular hypertrophy (LVH) due to long-standing AHT $[4,6]$. The pathogenesis of ventricular tachyarrhythmias in patients suffering from AHT is multifactorial, including hemodynamic and electrophysiological changes such as QT-prolongation $[2,7]$. Likewise, it could be speculated if an antihypertensive medication including thiazide diuretics might induce electrolyte shifts such as hypokalemia alleviating the onset of ventricular tachyarrhythmias $[2,8]$. The influence of AHT on the development of ventricular tachyarrhythmias is well described, whereas the prognostic impact of AHT in these high-risk patients is unclear.

Smoking of cigarettes is the most important modifiable risk factor for $\mathrm{CAD}$ [9]. The effect of smoking on progression of CAD is well established [3]. Nicotine by itself might induce acute vascular events due to induction of hypercoagulable states, induction of endothelial dysfunction, catecholamine release, and several toxic effects of increased carbon monoxide blood levels [10]. The role of smoking itself on ventricular tachyarrhythmias is still debated $[2,3]$. Nicotine has been proven to induce arrhythmogenesis in animals, whereas these findings have not yet been approved in humans [3]. However, there is no data available as to whether smoking has an impact on long-term mortality in patients presenting with ventricular tachyarrhythmias [3].

Therefore, the present study evaluates the prognostic impact of AHT and smoking on long-term all-cause mortality in patients presenting with ventricular tachyarrhythmias upon admission.

\section{Methods}

Study patients, design and data collection

The present study retrospectively included all consecutive patients with ventricular tachyarrhythmias from 2002 until 2016 at one institution. All relevant clinical data related to the index event was documented using patient files, daily records, documentation from diagnostic examinations and laboratory values, electrocardiograms (ECG), device recordings, and all further information derived from the electronic hospital information system.

Ventricular tachyarrhythmias are comprised of ventricular tachycardia (VT) and ventricular fibrillation (VF), as defined by current international guidelines [11]. Sustained VT was defined by VT with a duration of more than $30 \mathrm{~s}$ or causing hemodynamic collapse within $30 \mathrm{~s}$. Non-sustained VTs were defined as less than $30 \mathrm{~s}$. VTs were comprised of wide QRS complexes $(\geq 120 \mathrm{~ms}$ ) at a rate greater than $100 \mathrm{bpm}$ [11]. Ventricular tachyarrhythmias were documented by 12-lead ECG, ECG tele-monitoring and implantable cardioverter-defibrillators (ICD). In cases of unstable course or during cardiopulmonary resuscitation (CPR) documentation was performed by external defibrillator monitoring. Documented VF was treated by external defibrillation and in cases of prolonged instability with additional intravenous anti-arrhythmic drugs during CPR. Further documented data contained baseline characteristics, prior medical history, prior medical treatment, length of index stay, detailed findings of laboratory values at baseline, data derived from all non-invasive or invasive cardiac diagnostics and device therapies. These included coronary angiography, electrophysiological examination, prior or newly implanted ICDs, pacemakers or cardiac contractility modulators, which had already been implanted at index or at follow-up. Imaging modalities comprised echocardiography or cardiac magnetic resonance imaging (cMRI). The overall presence of an activated ICD summarizes the total sum of all patients with either a prior implanted ICD before admission, those undergoing new ICD implantation at index stay, as well as those with ICD implantation at the complete follow-up period after index hospitalization, referring to sole ICD, subcutaneous-ICD (s-ICD) and cardiac resynchronization therapy with defibrillator function (CRT-D). Pharmacological treatment was documented according to the discharge medication of patients surviving index hospitalization. Rates of overall 
ICDs and of pharmacological therapies refer to the number of surviving patients being discharged from index hospitalization.

The documentation period lasted from the index event until 2016. Documentation of all medical data was performed by independent cardiologists at the patients' individual period of hospitalization and were blinded to the final data analyses.

The present study is derived from an analysis of the "Registry of Malignant Arrhythmias and Sudden Cardiac Death-Influence of Diagnostics and Interventions (RACE-IT)" and represents a single-center registry including retrospectively consecutive patients presenting with ventricular tachyarrhythmias and SCD being acutely admitted to the University Medical Center Mannheim (UMM), Germany (clinicaltrials.gov identifier: NCT02982473) from 2002 until 2016. The registry was carried out according to the principles of the Declaration of Helsinki and was approved by the Medical Ethics Committee II of the Faculty of Medicine Mannheim, University of Heidelberg, Germany.

The medical center covers a general emergency department for emergency admission of traumatic, surgical, neurological and cardiovascular conditions. Interdisciplinary consultation is an inbuilt feature of this $24 / 7$ service, and is connected to a stroke unit, four intensive care units with extracorporeal life support and a chest pain unit to alleviate rapid triage of patients. The cardiology department itself includes $24 \mathrm{~h}$ catheterization and electrophysiologic laboratories, a hybrid operating room and telemetry units.

\section{Definition of study groups, inclusion and exclusion criteria}

For the present analysis risk stratification was performed according to AHT and smoking. AHT was defined by international guidelines as systolic blood pressure levels $\geq 140 \mathrm{mmHg}$ and/or diastolic blood pressure levels $\geq 90 \mathrm{mmHg}$, at which the benefits of treatment overweighed the risks of treatment, i.e. lifestyle modification or antihypertensive drugs [12]. Smoking was defined by daily nicotine use [13]. Overall exclusion criteria comprised patients with early cardiac death. Early cardiac death was defined as cardiac death occurring $<24 \mathrm{~h}$ after onset of ventricular tachyarrhythmias or an assumed unstable cardiac condition on index admission [11]. No further exclusion criteria were present.

Each patient was counted only once for inclusion when presenting with the first episode of ventricular tachyarrhythmias.

\section{Study endpoints}

The primary prognostic endpoint was all-cause mortality at long-term follow-up of 30 months. Overall follow-up lasted until 2016. All-cause mortality was documented using our electronic hospital information system and by directly contacting state resident registration offices ("bureau of mortality statistics") across Germany. Identification of patients was verified by place of name, surname, day of birth and registered living address. Lost to follow-up rate was $1.7 \%(\mathrm{n}=48)$ regarding survival until the end of the follow-up period.

\section{Statistical methods}

Quantitative data are presented as mean \pm standard error of mean (SEM), median and interquartile range (IQR), and ranges depending on the distribution of the data and were compared using the Student $t$ test for normally distributed data or the Mann-Whitney U test for nonparametric data. Deviations from a Gaussian distribution were tested by the Kolmogorov-Smirnov test. The Spearman rank correlation for nonparametric data was used to test univariate correlations. Qualitative data are presented as absolute and relative frequencies and were compared using the $\chi^{2}$ test or the Fisher exact test, as appropriate.

Uni-variable stratification was performed using the Kaplan-Meier method with comparisons between groups using uni-variable hazard ratios (HR) given together with 95\% confidence intervals applied in the propensity-matched cohorts.

Follow-up periods for evaluation of all-cause mortality was in accordance with the median survival of diseased patients to guarantee complete survival of at least $50 \%$ of patients. Patients not meeting long-term follow-up were censored.

The result of a statistical test was considered significant for $\mathrm{p}<0.05$, $\mathrm{p}$ values $\leq 0.01$ were defined as a statistical trend. SAS, release 9.4 (SAS Institute Inc., Cary, NC, USA) and SPSS (Version 25, IBM Armonk, New York, USA) were used for statistics.

\section{Propensity score matching}

Propensity matching was applied in advance (see study flow chart) and all statistical calculations as outlined above were performed in the propensity matched cohort afterwards.

In randomized controlled trials patients with or without a specific treatment would have a $50 \%$ chance of being treated and balanced measured and unmeasured baseline characteristics would be expected. However, patients cannot be rand- 
omized in real-life according to AHT and smoke. An observational study usually recruits consecutive real-life patients without randomization resulting in varying chances between $0 \%$ and $100 \%$ to receive imbalances in baseline characteristics and treatments. Therefore, differences inoutcomes of specific groups might be explained by heterogeneous distribution of baseline characteristics and applied therapies. To further reduce this selection bias, a 1:1 propensity-scores were used for AHT vs. no AHT and smokers vs. non-smokers, to assemble matched cohorts, in which patients were well-balanced regarding all measured baseline characteristics. $1: 1$ propensity score matching was performed and included the entire study cohort $[14,15]$.

Propensity scores were created according to the presence of the following independent variables: age, gender, diabetes, chronic kidney disease (CKD) (glomerular filtration rate $<60 \mathrm{~mL} / \mathrm{min} / 1.73 \mathrm{~m}^{2}$ ), $\mathrm{CAD}$, left ventricular dysfunction, $\mathrm{CPR}$, acute myocardial infarction (AMI), cardiogenic shock, presence of an ICD and index ventricular tachyarrhythmias (VT vs. VF).

Based on propensity score values counted by logistic regression, for each AHT a non-AHT patient and for each smoking patient, a non-smoking patient with a similar propensity score value was found (accepted difference of propensity score value $<5 \%)$.

\section{Results}

\section{Study population}

Propensity-matched cohorts of consecutive patients presenting with ventricular tachyarrhythmias on admission at our institution consisted of a total of 988 patients in AHT and 872 patients in smoking cohorts (Fig. 1).

In the AHT cohort (494 patients in each group), the rate of VT and VF was similar in both groups (VT: AHT 60\% vs. no AHT 60\%; VF: AHT 40\% vs. no AHT 40\%) (Table 1). No further differences were observed for prognosis-relevant factors at index including CKD, CAD, AMI, cardiogenic shock, cardiomyopathy as well as discharge medication with beta-blockers, angiotensin-converting enzyme inhibitors (ACEI) and aldosterone antagonists in between AHT and no AHT patients (Table 1).

In the smoking cohort (436 patients in each group), the rate of VT and VF was similar in both groups (VT: smokers $61 \%$ vs. non-smokers $62 \%$; VF: smokers $39 \%$ vs. non-smokers $38 \%$ ) (Table 2). Despite matching for prognosis-relevant factors

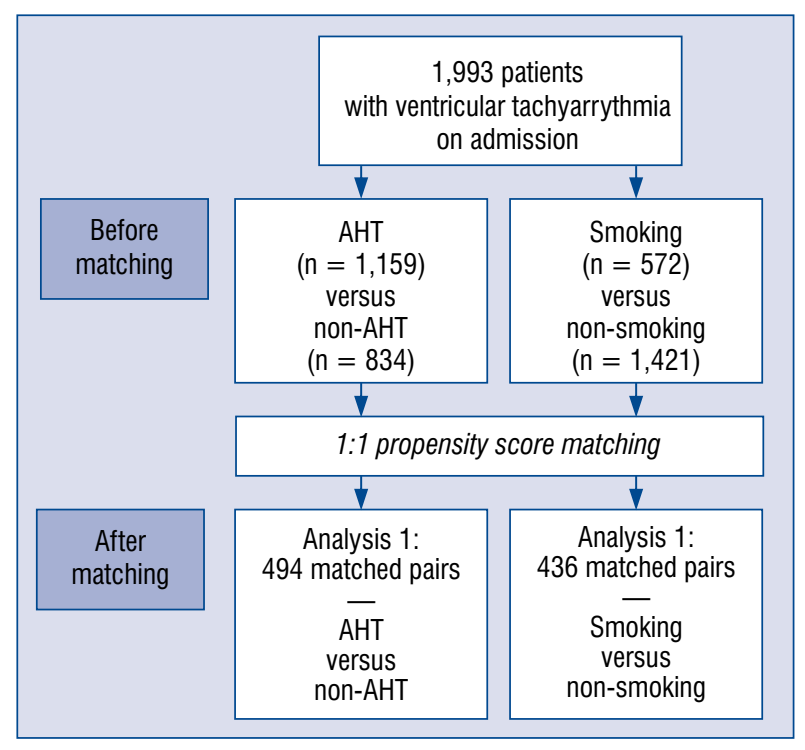

Figure 1. Flow chart of study population; AHT — arterial hypertension.

at index including $\mathrm{CKD}, \mathrm{CAD}$, AMI, cardiogenic shock, cardiomyopathies and atrial fibrillation, smokers were still more likely to have a prior history of AHT, hyperlipidemia, cardiac family history and ACEI at discharge (Table 2).

The AHT cohort and the smoker's cohort were well matched for left ventricular function, cardiac therapies at index including CPR, diagnostics including coronary angiography and CAD status and device therapies (Tables 1,2). Smokers underwent coronary angiography more often and revealed a higher rate of overall CAD.

\section{All-cause mortality and survival data}

Paradoxically, at long-term follow-up high-risk AHT patients presenting with ventricular tachyarrhythmias were not associated with the primary endpoint of long-term all-cause mortality at 30 months (IQR 96 days - 5.5 years) compared to no AHT patients (mortality rates: AHT, 26\% vs. no AHT: 28\%; log-rank p $=0.525)$ (Fig. 2).

Arterial hypertension was not associated with the primary endpoint of long-term all-cause mortality in CRT (HR 0.511 [0.132-1.976]; p = 0.330) and no-CRT patients (HR 0.942 [0.736-1.205]; $\mathrm{p}=0.942$ ) as well as in patients with left ventricular ejection fraction (LVEF) $>35 \%$ and LVEF $<35 \%$ $(\mathrm{LVEF}>35 \%$, log-rank $\mathrm{p}=0.497 ; \mathrm{LVEF}<35 \%$, $\log$-rank $\mathrm{p}=0.753$ ) (data not shown).

Accordingly, smokers presenting with ventricular tachyarrhythmias on admission were not associated with the primary endpoint of all-cause 
Table 1. Characteristics of matched patients with or without arterial hypertension.

\begin{tabular}{|c|c|c|c|c|c|}
\hline \multirow{2}{*}{$\begin{array}{l}\text { Characteristic } \\
\text { Gender: male }\end{array}$} & \multicolumn{2}{|c|}{$\begin{array}{c}\text { Non-AHT } \\
\text { (n = 494; } 50 \%)\end{array}$} & \multicolumn{2}{|c|}{$\begin{array}{c}\text { AHT } \\
\text { (n }=494 ; 50 \%)\end{array}$} & \multirow{2}{*}{$\begin{array}{c}P \\
0.881\end{array}$} \\
\hline & 376 & $(76 \%)$ & 378 & $(77 \%)$ & \\
\hline Age, median (range) & \multicolumn{2}{|c|}{$65(21-89)$} & \multicolumn{2}{|c|}{$65(30-94)$} & 0.396 \\
\hline \multicolumn{6}{|l|}{ Ventricular tachyarrhythmias: } \\
\hline VT & 296 & $(60 \%)$ & 298 & $(60 \%)$ & \multirow{2}{*}{0.897} \\
\hline VF & 198 & $(40 \%)$ & 196 & $(40 \%)$ & \\
\hline \multicolumn{6}{|l|}{ Cardiovascular risk factors: } \\
\hline Diabetes mellitus & 90 & $(18 \%)$ & 115 & $(23 \%)$ & 0.050 \\
\hline Hyperlipidemia & 74 & $(15 \%)$ & 211 & $(43 \%)$ & 0.001 \\
\hline Smoking & 140 & $(28 \%)$ & 160 & $(32 \%)$ & 0.166 \\
\hline Cardiac family history & 52 & $(11 \%)$ & 63 & $(13 \%)$ & 0.275 \\
\hline \multicolumn{6}{|l|}{ Comorbidities at index stay: } \\
\hline Acute myocardial infarction & 161 & $(33 \%)$ & 145 & $(29 \%)$ & 0.271 \\
\hline Cardiogenic shock & 58 & $(12 \%)$ & 59 & $(12 \%)$ & 0.922 \\
\hline Cardiopulmonary resuscitation & 187 & $(38 \%)$ & 177 & $(36 \%)$ & 0.510 \\
\hline Cardiomyopathy & 78 & $(16 \%)$ & 60 & $(12 \%)$ & 0.099 \\
\hline Valvular heart disease & 44 & $(9 \%)$ & 42 & $(9 \%)$ & 0.821 \\
\hline Atrial fibrillation & 137 & $(28 \%)$ & 173 & $(35 \%)$ & 0.014 \\
\hline Cardiac surgery & 7 & $(1 \%)$ & 7 & $(1 \%)$ & 1.000 \\
\hline Hyperkalemia & 7 & $(1 \%)$ & 8 & $(2 \%)$ & 0.795 \\
\hline Hypokalemia & 28 & $(6 \%)$ & 34 & $(7 \%)$ & 0.431 \\
\hline Stroke & 15 & $(3 \%)$ & 19 & $(4 \%)$ & 0.485 \\
\hline Clinically significant bleeding & 18 & $(4 \%)$ & 16 & $(3 \%)$ & 0.727 \\
\hline Anemia & 24 & $(5 \%)$ & 31 & $(6 \%)$ & 0.331 \\
\hline Septic shock & 13 & $(3 \%)$ & 13 & $(3 \%)$ & 1.000 \\
\hline Chronic kidney disease & 217 & $(44 \%)$ & 219 & $(44 \%)$ & 0.898 \\
\hline Liver cirrhosis & 7 & $(1 \%)$ & 4 & $(0.8 \%)$ & 0.363 \\
\hline \multicolumn{6}{|l|}{ Coronary angiography: } \\
\hline Coronary angiography, overall & 337 & $(68 \%)$ & 368 & $(75 \%)$ & 0.029 \\
\hline Coronary artery disease & 243 & $(72 \%)$ & 254 & $(69 \%)$ & 0.130 \\
\hline No evidence of CAD: & 94 & $(28 \%)$ & 114 & $(31 \%)$ & 0.163 \\
\hline 1-vessel & 78 & $(23 \%)$ & 100 & $(27 \%)$ & 0.069 \\
\hline 2-vessel & 88 & $(26 \%)$ & 70 & $(19 \%)$ & 0.118 \\
\hline 3-vessel & 77 & $(23 \%)$ & 84 & $(23 \%)$ & 0.547 \\
\hline Presence of CABG & 37 & $(11 \%)$ & 44 & $(12 \%)$ & 0.684 \\
\hline Presence of СТO & 71 & $(21 \%)$ & 60 & $(16 \%)$ & 0.085 \\
\hline $\mathrm{PCl}$ & 157 & $(47 \%)$ & 138 & $(38 \%)$ & 0.015 \\
\hline Left ventricular function: & & & & & 0.786 \\
\hline LVEF > 55\% & 143 & $(29 \%)$ & 150 & $(30 \%)$ & \\
\hline LVEF $54-35 \%$ & 176 & $(36 \%)$ & 162 & $(33 \%)$ & \\
\hline LVEF $<35 \%$ & 175 & $(35 \%)$ & 182 & $(37 \%)$ & \\
\hline Patients at discharge & 426 & $(86 \%)$ & 442 & $(89 \%)$ & 0.119 \\
\hline Overall ICDs & 131 & $(31 \%)$ & 132 & $(30 \%)$ & 0.911 \\
\hline \multicolumn{6}{|l|}{ Medication at discharge: } \\
\hline Beta-blocker & 358 & $(84 \%)$ & 379 & $(86 \%)$ & 0.482 \\
\hline ACEI & 290 & $(68 \%)$ & 314 & $(71 \%)$ & 0.342 \\
\hline Alosterone antagonist & 48 & $(11 \%)$ & 58 & $(13 \%)$ & 0.404 \\
\hline
\end{tabular}


Table 1. (cont.). Characteristics of matched patients with or without arterial hypertension.

\begin{tabular}{|c|c|c|c|c|c|}
\hline Characteristic & \multicolumn{2}{|c|}{$\begin{array}{c}\text { Non-AHT } \\
(\mathrm{n}=494 ; 50 \%)\end{array}$} & \multicolumn{2}{|c|}{$\begin{array}{c}\text { AHT } \\
(n=494 ; 50 \%)\end{array}$} & $\mathbf{P}$ \\
\hline \multicolumn{6}{|c|}{ Hospitalization time [days], median (IQR): } \\
\hline Total hospitalization time & \multicolumn{2}{|c|}{$13(8-22)$} & \multicolumn{2}{|c|}{$14(8-23)$} & 0.991 \\
\hline ICU time & \multicolumn{2}{|c|}{$4(0-8)$} & \multicolumn{2}{|c|}{$3(0-8)$} & 0.278 \\
\hline $\begin{array}{l}\text { Follow-up time [days], } \\
\text { mean; median (range) }\end{array}$ & \multicolumn{2}{|c|}{$\begin{array}{c}1719 ; 1539 \\
(3-5095)\end{array}$} & \multicolumn{2}{|c|}{$\begin{array}{c}1809 ; 1595 \\
(3-5106)\end{array}$} & 0.331 \\
\hline \multicolumn{6}{|c|}{ All-cause mortality at 30 months: } \\
\hline At index & 68 & $(14 \%)$ & 52 & $(11 \%)$ & 0.119 \\
\hline At follow up & 68 & $(14 \%)$ & 76 & $(15 \%)$ & 0.471 \\
\hline Overall & 136 & $(28 \%)$ & 128 & $(26 \%)$ & 0.377 \\
\hline
\end{tabular}

ACEI - angiotensin converting enzyme inhibitor; AHT - arterial hypertension; AMI - acute myocardial infarction; CABG - coronary artery bypass grafting; CAD - coronary artery disease; CTO - coronary chronic total occlusion; ICD - implantable cardioverter-defibrillator; ICU - intensive care unit; IQR — interquartile range; LVEF — left ventricular ejection function; $\mathrm{PCI}$ - percutaneous coronary intervention; VF ventricular fibrillation; VT - ventricular tachycardia

Table 2. Baseline characteristics according to smoking status.

\begin{tabular}{|c|c|c|c|c|c|}
\hline \multirow{2}{*}{$\begin{array}{l}\text { Characteristic } \\
\text { Gender: male }\end{array}$} & \multicolumn{2}{|c|}{$\begin{array}{l}\text { Non-smoking } \\
(\mathrm{n}=436 ; 50 \%)\end{array}$} & \multicolumn{2}{|c|}{$\begin{array}{c}\text { Smoking } \\
(\mathrm{n}=436 ; 50 \%)\end{array}$} & \multirow{2}{*}{$\begin{array}{c}\mathbf{P} \\
0.785\end{array}$} \\
\hline & 362 & $(83 \%)$ & 365 & $(84 \%)$ & \\
\hline Age, median (range) & \multicolumn{2}{|c|}{$65(16-92)$} & \multicolumn{2}{|c|}{$63(21-88)$} & 0.001 \\
\hline \multicolumn{6}{|l|}{ Ventricular tachyarrhythmias: } \\
\hline VT & 269 & $(62 \%)$ & 266 & $(61 \%)$ & \multirow{2}{*}{0.835} \\
\hline VF & 167 & $(38 \%)$ & 170 & $(39 \%)$ & \\
\hline \multicolumn{6}{|l|}{ Cardiovascular risk factors: } \\
\hline Arterial hypertension & 252 & $(58 \%)$ & 293 & $(67 \%)$ & 0.004 \\
\hline Diabetes mellitus & 113 & $(26 \%)$ & 110 & $(25 \%)$ & 0.816 \\
\hline Hyperlipidemia & 135 & $(31 \%)$ & 180 & $(41 \%)$ & 0.002 \\
\hline Cardiac family history & 33 & $(8 \%)$ & 85 & $(20 \%)$ & 0.001 \\
\hline \multicolumn{6}{|l|}{ Comorbidities at index: } \\
\hline Acute myocardial infarction & 132 & $(30 \%)$ & 155 & $(36 \%)$ & 0.097 \\
\hline Cardiogenic shock & 39 & $(9 \%)$ & 56 & $(13 \%)$ & 0.065 \\
\hline Cardiomyopathy & 47 & $(11 \%)$ & 36 & $(8 \%)$ & 0.204 \\
\hline Cardiopulmonary resuscitation & 156 & $(36 \%)$ & 156 & $(36 \%)$ & 1.000 \\
\hline Valvular heart disease & 30 & $(7 \%)$ & 26 & $(6 \%)$ & 0.581 \\
\hline Atrial fibrillation & 120 & $(28 \%)$ & 123 & $(28 \%)$ & 0.821 \\
\hline Cardiac surgery & 4 & $(0.9 \%)$ & 15 & $(3 \%)$ & 0.011 \\
\hline Hyperkalemia & 3 & $(0.7 \%)$ & 6 & $(1 \%)$ & 0.315 \\
\hline Hypokalemia & 31 & $(7 \%)$ & 16 & $(4 \%)$ & 0.024 \\
\hline Stroke & 8 & $(2 \%)$ & 19 & $(4 \%)$ & 0.032 \\
\hline Clinically significant bleeding & 6 & $(1 \%)$ & 15 & $(3 \%)$ & 0.047 \\
\hline Anemia & 19 & $(4 \%)$ & 21 & $(5 \%)$ & 0.746 \\
\hline Septic shock & 5 & $(1 \%)$ & 13 & $(3 \%)$ & 0.057 \\
\hline Chronic kidney disease & 175 & $(40 \%)$ & 173 & $(40 \%)$ & 0.890 \\
\hline Liver cirrhosis & 3 & $(0.7 \%)$ & 6 & $(1 \%)$ & 0.315 \\
\hline \multicolumn{6}{|l|}{ Coronary angiography: } \\
\hline Coronary angiography, overall & 297 & $(68 \%)$ & 328 & $(75 \%)$ & 0.020 \\
\hline Coronary artery disease & 234 & $(54 \%)$ & 263 & $(60 \%)$ & 0.047 \\
\hline
\end{tabular}


Table 2 (cont.). Baseline characteristics according to smoking status.

\begin{tabular}{|c|c|c|c|c|c|}
\hline \multirow{2}{*}{$\begin{array}{l}\text { Characteristic } \\
\text { No evidence of CAD: }\end{array}$} & \multicolumn{2}{|c|}{$\begin{array}{l}\text { Non-smoking } \\
(n=436 ; 50 \%)\end{array}$} & \multicolumn{2}{|c|}{$\begin{array}{c}\text { Smoking } \\
(\mathrm{n}=436 ; 50 \%)\end{array}$} & \multirow{2}{*}{$\begin{array}{c}P \\
0.837\end{array}$} \\
\hline & 63 & $(21 \%)$ & 65 & $(20 \%)$ & \\
\hline 1-vessel & 73 & $(25 \%)$ & 97 & $(30 \%)$ & 0.040 \\
\hline 2-vessel & 81 & $(27 \%)$ & 71 & $(22 \%)$ & 0.797 \\
\hline 3-vessel & 80 & $(27 \%)$ & 95 & $(29 \%)$ & 0.205 \\
\hline Presence of CABG & 45 & $(15 \%)$ & 44 & $(13 \%)$ & 0.535 \\
\hline Presence of CTO & 69 & $(23 \%)$ & 68 & $(21 \%)$ & 0.450 \\
\hline $\mathrm{PCl}$ & 123 & $(41 \%)$ & 157 & $(48 \%)$ & 0.105 \\
\hline Left ventricular function: & & & & & 0.679 \\
\hline LVEF > 55\% & 126 & $(29 \%)$ & 125 & $(29 \%)$ & \\
\hline LVEF 54-35\% & 153 & $(35 \%)$ & 152 & $(35 \%)$ & \\
\hline LVEF $<35 \%$ & 157 & $(36 \%)$ & 159 & $(37 \%)$ & \\
\hline Patients at discharge & 399 & $(91 \%)$ & 389 & $(89 \%)$ & 0.181 \\
\hline Overall ICDs & 115 & $(29 \%)$ & 124 & $(32 \%)$ & 0.467 \\
\hline \multicolumn{6}{|l|}{ Medication at discharge: } \\
\hline Beta-blocker & 338 & $(85 \%)$ & 341 & $(88 \%)$ & 0.231 \\
\hline ACEI & 257 & $(64 \%)$ & 286 & $(74 \%)$ & 0.006 \\
\hline Aldosterone antagonist & 32 & $(8 \%)$ & 47 & $(12 \%)$ & 0.058 \\
\hline \multicolumn{6}{|c|}{ Hospitalization time [days], median (IQR): } \\
\hline Total hospitalization time & \multicolumn{2}{|c|}{$12(4-23)$} & \multicolumn{2}{|c|}{$13(8-23)$} & 0.261 \\
\hline ICU time & \multicolumn{2}{|c|}{$3(0-8)$} & \multicolumn{2}{|c|}{$3(0-8)$} & 0.383 \\
\hline $\begin{array}{l}\text { Follow-up time [days], } \\
\text { mean; median (range) }\end{array}$ & \multicolumn{2}{|c|}{$\begin{array}{l}1954 ; 1930 \\
(3-5095)\end{array}$} & \multicolumn{2}{|c|}{$\begin{array}{c}1693 ; 1527 \\
(3-5089)\end{array}$} & 0.006 \\
\hline \multicolumn{6}{|c|}{ All-cause mortality at 30 months: } \\
\hline At index & 37 & $(9 \%)$ & 47 & $(11 \%)$ & 0.191 \\
\hline At follow up & 71 & $(16 \%)$ & 51 & $(12 \%)$ & 0.051 \\
\hline Overall & 108 & $(25 \%)$ & 98 & $(22 \%)$ & 0.425 \\
\hline
\end{tabular}

ACEI - angiotensin converting enzyme inhibitor; AHT — arterial hypertension; AMI - acute myocardial infarction; CABG - coronary artery bypass grafting; CAD — coronary artery disease; CTO — coronary chronic total occlusion; ICD - implantable cardioverter-defibrillator; ICU - intensive care unit; IQR — interquartile range; LVEF — left ventricular ejection function; $\mathrm{PCI}$ - percutaneous coronary intervention; VF - ventricular fibrillation; VT — ventricular tachycardia

mortality at 30 months compared to non-smokers (mortality rate smokers: $22 \%$ vs. non-smokers: $25 \%$; log-rank $\mathrm{p}=0.683$ ) (Fig. 3). Even in patients with LVEF > 35\% smoking was not associated with any mortality difference (LVEF > 35\%, log-rank $\mathrm{p}=0.649 ; \mathrm{LVEF}<35 \%$, log-rank $\mathrm{p}=0.239)($ data not shown).

\section{Discussion}

The present study evaluates the prognostic impact of AHT and smoking on long-term allcause mortality in high-risk patients presenting consecutively with ventricular tachyarrhythmias on admission. Paradoxically, this well-matched analysis suggests that neither AHT nor smoking were associated with increased secondary long- term all-cause mortality at 30 months in patients presenting consecutively with ventricular tachyarrhythmias straight from the admission scenario.

Previous studies showed that LVH due to AHT is a risk factor for ventricular tachyarrhythmias [16]. Data from the Framingham cohort demonstrated in 1985, that AHT may increase the risk of sustained or non-sustained VT in the presence of LVH [17]. Depending on the grade of LVH, the authors showed an increasing risk of cardiovascular and death of any cause. However, this study did not report on a long-term follow up of patients presenting on admission with ventricular tachyarrhythmias according to the presence of AHT. The present study did not prove any prognostic impact of AHT on long-term prognosis in patients presenting with ventricular tachyarrhythmias. Notably, no prog- 


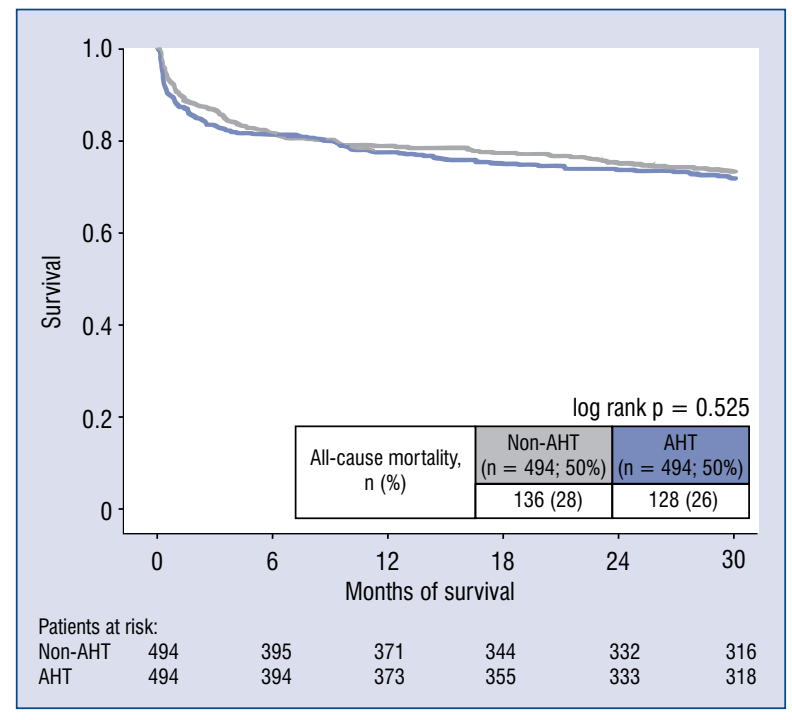

Figure 2. Long-term all-cause mortality of patients presenting with ventricular tachyarrhythmias stratified according to the presence and absence of arterial hypertension (AHT).

nostic impact of AHT was additionally observed in patients with or without CRT devices.

McLenachan et al. [18] compared patients with and without LVH and AHT assessed by 12-lead ECG (each $\mathrm{n}=50$ ) including matching for age, sex, smoking and blood-pressure levels. It was demonstrated that AHT/LVH patients had a significant higher rate of ventricular tachyarrhythmias and SCD compared to patients without LVH [18]. Furthermore, effective control of blood pressure was shown to prevent ventricular tachyarrhythmias and SCD [16]. Especially, antihypertensive drugs, such as thiazide diuretics, were associated with ventricular tachyarrhythmias due to electrolyte shifts. Although, the exact mechanism is not fully understood, hypokalemia might play an important role [19]. In the double-blind and randomized HOPE-study the ACEI ramipril was associated with a reduced rate of death, myocardial infarction, stroke and cardiac arrest compared to placebo [20]. Since their market approval in the early 1980s, ACEI became the international gold standard in the therapy of AHT. ACEI are known to attenuate adverse structural myocardial remodeling and progression of LVH [21-23]. Data from Framingham and MCLenachan were collected until 1985/1987, whereas data about ACEI treatment were not reported sufficiently. It might be speculated as to whether the lack of prognostic impact of AHT itself may be attributed to modern therapies including ACEI within 2002 and 2016. In this study 26\%

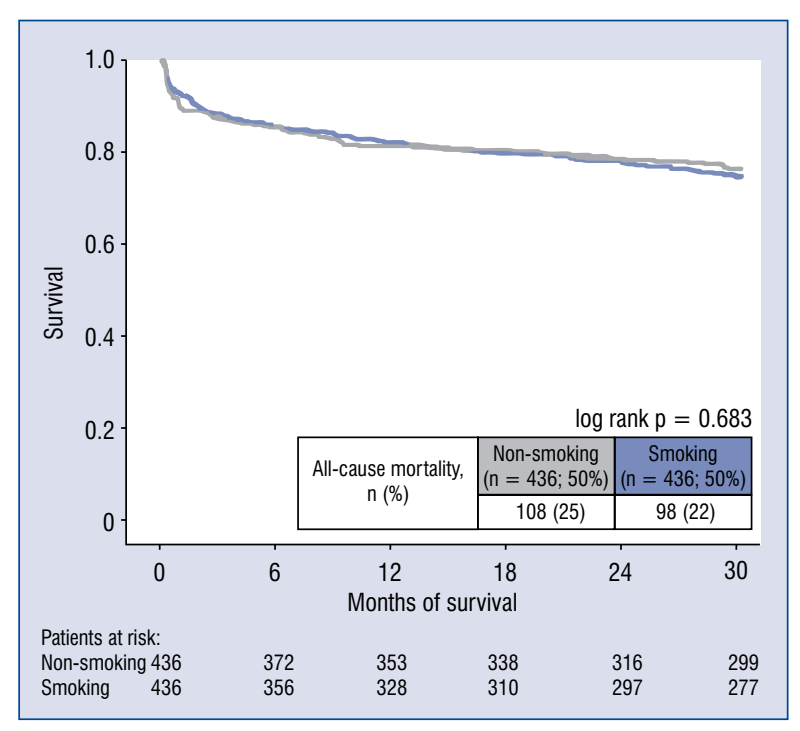

Figure 3. Long-term all-cause mortality of patients presenting with ventricular tachyarrhythmias stratified according to the presence and absence of smoking.

of AHT patients had been already treated with ACEI.

A majority of patients included in the present study suffered from systolic HF (LVEF $<55 \%)$ in almost two thirds of cases. Heart failure, especially in advanced stages ( $\mathrm{LVEF}<35 \%)$ is accompanied by progressive AHT in terms of low-output failure [24]. This might additionally support the hypothesis of risk-factor paradox in patients presenting with ventricular tachyarrhythmias related to AHT. Here, the implantation of CRT-devices represents an established treatment option in patients with wide QRS complexes and LVEF < 35\% [25, 26], and aims to improve left ventricular dysfunction by an effective biventricular stimulation rate of $>98 \%$, which in turn, may increase systolic blood pressure. However, there are known predictors of ineffective CRT response, such as permanent atrial fibrillation, massively dilated left ventricles, severe mitral regurgitation, and large ischemic anteroseptal or posterior scars of the left ventricle [26]. In order to test for potential bias, the present study demonstrates that AHT had no effect on prognosis and neither in CRT, non-CRT and LVEF $<35 \%$ patients. However, the outlined circumstances might additionally support the hypothesis of the entitled risk-factor paradox in patients presenting with ventricular tachyarrhythmias related to AHT.

The only and most modifiable cardiovascular risk factor represents the smoking of cigarettes, however smoking is still the leading preventable 
cause of death in the United States [3]. Smoking is directly associated with atherosclerosis, whereas its influence in patients with ventricular tachyarrhythmias is still unclear [3]. Several experimental studies investigated the toxicity of nicotine in animals and cell cultures. Mehta et al. [27] investigated the effect of intravenous application of nicotine in healthy dogs. In their dose-response study, increased rates of arrhythmias were observed at a dosage of $50 \mu \mathrm{g} / \mathrm{kg}$ (equivalent in humans of 2 smoked cigarettes), including supraventricular, atrioventricular junctional and ventricular arrhythmias [27]. An intravenous dosage of nicotine at $100 \mu \mathrm{g} / \mathrm{kg}$ was shown to induce fatal ventricular flutter and fibrillation. The arrhythmogenic side effect of nicotine was explained by increased carbon monoxide and oxidative stress [27]. Unfortunately, these experimental results are not directly comparable to humans, due to long-term use of smoking in humans $[3,27]$.

In a case-control study including 95 CAD patients undergoing bypass surgery smoking was an independent predictor of atrial fibrosis [28]. Goette et al. [28] demonstrated increased levels of collagen type III within an organo-typical atrial tissue culture model of smokers. This pro-fibrotic status (i.e. increase of extracellular matrix turnover and subsequent adverse structural myocardial remodeling) as a consequence of nicotine was also shown in an experimental study in dogs by Shan et al. [29], where a dose-dependent increase of atrial remodeling after intravenous administration of nicotine over 30 days was documented. Atrial tissue was preserved during open-heart surgery of the dogs and the fibrous tissue was finally quantified microscopically [3]. Furthermore, arrhythmogenesis might be related to tissue hypoxemia, oxidative stress and increased carbon monoxide by binding hemoglobin during smoking [30, 31].

Plank et al. [32] investigated in a sub-study of the Multicenter Automatic Defibrillator Implantation Trial-Cardiac Resynchronization Therapy (MADIT-CRT-trial) the risk of first and recurrent ventricular tachyarrhythmias in patients with mild HF and ICD therapy according to smoking [32]. The study compared 465 non-smokers, 780 past smokers and 197 current smokers with ischemic and non-ischemic cardiomyopathy [32]. Current smokers with mild HF were shown to be associated with higher risk of ventricular tachyarrhythmias compared to past- and non-smokers [32]. The MADIT-II trial showed that the risk of ventricular tachyarrhythmias in patients with CAD-related HF is higher in current smokers compared to past- and non-smokers. They also showed that smokers had a significantly higher risk for inadequate ICD-shocks [33]). MADIT-II and MADIT-CRT were primary preventive studies, whereas the present study included patients who already survived an episode of ventricular tachyarrhythmias on admission. Unfortunately, sub-analyses of MADIT did not undergo propensity matching which questions the results due to cofounding of heterogeneous sub-groups regarding comorbidities.

Data about the effect of nicotine and cardiac arrhythmia are limited in humans. Pharmacodynamics of nicotine is complex including the biphasic dose-response curve and additional development of nicotine tolerance. Therefore, more studies in humans are necessary to prove potential acute toxic and long-term prognostic effects of nicotine in patients presenting with ventricular tachyarrhythmias [3, 34-36]. Consistently, smoking does represent a risk factor for development of CAD and chronic obstructive pulmonary disease (COPD). Both diseases might promote ventricular tachyarrhythmias by themselves [37]. No evidence is yet available whether smoking may affect prognosis in the absence of CAD and COPD, even in high-risk patients with ventricular tachyarrhythmias.

In conclusion, this study demonstrates a paradox that neither AHT nor smoking reveal any impact on long-term prognosis in terms of all-cause mortality in high-risk patients presenting with ventricular tachyarrhythmias on admission.

\section{Limitations of the study}

This observational and retrospective registrybased analysis reflects a realistic picture of consecutive health-care supply of high-risk patients presenting with ventricular tachyarrhythmias right upon hospital admission. Lost to follow-up rate regarding the evaluated endpoint of all-cause mortality was minimal. Additionally, heterogeneity within the study population was controlled by 1:1 propensity-matched analyses. The potential influence of optimal treatment of hypertensive patients as well as investigation of smoking cessation programmes being offered at the documented institution might still have influenced the present results to a minor extent. Despite retrospective propensity-score matching with similar rates of cardioprotective drugs in both AHT and no AHT patients and no differences after stratification into left ventricular dysfunction, a certain bias may still be present especially in hypotensive patients associated with progressive $\mathrm{HF}$ usually receiving minor dosages and numbers of cardioprotective 
drugs. Also, a CRT-treatment might improve the blood pressure and consecutive the survival in hypotensive patients. The prognostic impact of AHT and smoking in patients with ventricular tachyarrhythmias has to be re-evaluated in further studies.

\section{Conclusions}

Paradoxically, AHT and smoking were not associated with increased long-term all-cause mortality at 30 months in patients presenting with ventricular tachyarrhythmias on admission.

Funding: Supported by the DZHK (Deutsches Zentrum fuer Herz-Kreislauf-Forschung - German Centre for Cardiovascular Research).

\section{Conflict of interest: None declared}

\section{References}

1. Green D, Roberts P, New D, et al. Sudden cardiac death in hemodialysis patients: an in-depth review. Am J Kidney Dis. 2011; 57(6): 921-929, doi: 10.1053/j.ajkd.2011.02.376.

2. Lip G, Coca A, Kahan T, et al. Hypertension and cardiac arrhythmias: a consensus document from the European Heart Rhythm Association (EHRA) and ESC Council on Hypertension, endorsed by the Heart Rhythm Society (HRS), Asia-Pacific Heart Rhythm Society (APHRS) and Sociedad Latinoamericana de Estimulación Cardíaca y Electrofisiología (SOLEACE). EP Europace. 2017; 19(6): 891-911, doi: 10.1093/europace/eux091.

3. D’Alessandro A, Boeckelmann I, Hammwhöner M, et al. Nicotine, cigarette smoking and cardiac arrhythmia: an overview. Eur J Prev Cardiol. 2012; 19(3): 297-305, doi: 10.1177/1741826711411738, indexed in Pubmed: 22779085.

4. Lip GYH, Coca A, Kahan T, et al. Hypertension and cardiac arrhythmias: executive summary of a consensus document from the European Heart Rhythm Association (EHRA) and ESC Council on Hypertension, endorsed by the Heart Rhythm Society (HRS), Asia-Pacific Heart Rhythm Society (APHRS), and Sociedad Latinoamericana de Estimulación Cardíaca y Electrofisiología (SOLEACE). Eur Heart J Cardiovasc Pharmacother. 2017; 3(4): 235-250, doi: 10.1093/ehjcvp/pvx019, indexed in Pubmed: 28541499.

5. Aronow WS, Epstein S, Koenigsberg M, et al. Usefulness of echocardiographic abnormal left ventricular ejection fraction, paroxysmal ventricular tachycardia and complex ventricular arrhythmias in predicting new coronary events in patients over 62 years of age. Am J Cardiol. 1988; 61(15): 1349-1351, indexed in Pubmed: 3376895.

6. Levy D, Anderson KM, Savage DD, et al. Risk of ventricular arrhythmias in left ventricular hypertrophy: the Framingham Heart Study. Am J Cardiol. 1987; 60(7): 560-565, indexed in Pubmed: 2957907.

7. Sultana R, Sultana N, Rashid A, et al. Cardiac arrhythmias and left ventricular hypertrophy in systemic hypertension. J Ayub Med Coll Abbottabad. 2010; 22(4): 155-158, indexed in Pubmed: 22455286.
8. Roush GC, Sica DA. Diuretics for hypertension: a review and update. Am J Hypertens. 2016; 29(10): 1130-1137, doi: 10.1093/ ajh/hpw030, indexed in Pubmed: 27048970.

9. Services UDoHaH. The 2004 United States Surgeon General's Report: The Health Consequences of Smoking. N S W Public Health Bull. 2004;15(5-6). 107.

10. Benowitz NL, Hansson A, Jacob P. Cardiovascular effects of nasal and transdermal nicotine and cigarette smoking. Hypertension. 2002; 39(6): 1107-1112, indexed in Pubmed: 12052850.

11. Priori SG, Blomstrom-Lundqvist C, Mazzanti A, et al. 2015 ESC Guidelines for the management of patients with ventricular arrhythmias and the prevention of sudden cardiac death: The Task Force for the Management of Patients with Ventricular Arrhythmias and the Prevention of Sudden Cardiac Death of the European Society of Cardiology (ESC). Endorsed by: Association for European Paediatric and Congenital Cardiology (AEPC). Eur Heart J. 2015; 36(41): 2793-867.

12. Williams B, Mancia G, Spiering W, et al. 2018 ESC/ESH Guidelines for the management of arterial hypertension. Eur Heart J. 2018; 39(33): 3021-3104, doi: 10.1093/eurheartj/ehy339, indexed in Pubmed: 30165516.

13. Wienbergen H, Hambrecht R. [ESC-Guidelines 2016 - Prevention of Cardiovascular Diseases in Clinical Practice]. Dtsch Med Wochenschr. 2017; 142(3): 189-192, doi: 10.1055/s-0042-113928, indexed in Pubmed: 28187483.

14. Ferdinand D, Otto M, Weiss C. Get the most from your data: a propensity score model comparison on real-life data. Int J Gen Med. 2016; 9: 123-131, doi: 10.2147/JJGM.S104313, indexed in Pubmed: 27274306.

15. Austin PC. An introduction to propensity score methods for reducing the effects of confounding in observational studies. Multivariate Behav Res. 2011; 46(3): 399-424, doi: 10.1080/002 73171.2011.568786, indexed in Pubmed: 21818162.

16. Kannel WB, McGee DL. Epidemiology of sudden death: insights from the Framingham Study. Cardiovasc Clin. 1985; 15(3): 93-105, indexed in Pubmed: 3833369.

17. Levy D, Garrison R, Savage D, et al. Prognostic Implications of Echocardiographically Determined Left Ventricular Mass in the Framingham Heart Study. N Engl J Med. 1990; 322(22): 1561-1566, doi: 10.1056/nejm199005313222203.

18. McLenachan JM, Henderson E, Morris KI, et al. Ventricular arrhythmias in patients with hypertensive left ventricular hypertrophy. N Engl J Med. 1987; 317(13): 787-792, doi: 10.1056/ NEJM198709243171302, indexed in Pubmed: 2957590.

19. Siscovick DS, Raghunathan TE, Psaty BM, et al. Diuretic therapy for hypertension and the risk of primary cardiac arrest. N Engl J Med. 1994; 330(26): 1852-1857, doi: 10.1056/ NEJM199406303302603, indexed in Pubmed: 8196728.

20. Correction: Effects of An Angiotensin-Converting-Enzyme Inhibitor, Ramipril, on Cardiovascular Events in High-Risk Patients. N Engl J Med. 2000; 342(18): 1376, indexed in Pubmed: 10706908.

21. Mancia G, Fagard R, Narkiewicz K, et al. Task Force Members. $2013 \mathrm{ESH} / \mathrm{ESC}$ Guidelines for the management of arterial hypertension: the Task Force for the management of arterial hypertension of the European Society of Hypertension (ESH) and of the European Society of Cardiology (ESC). J Hypertens. 2013; 31(7): 1281-1357, doi: 10.1097/01.hjh.0000431740.32696.cc, indexed in Pubmed: 23817082.

22. Captopril--a decade of discovery. Proceedings of a symposium. London, 24-25 October 1985. Postgrad Med J. 1986; 62 Suppl 1: 1-191, indexed in Pubmed: 3534844. 
23. Smith CG, Vane JR, Smith CG, et al. The discovery of captopril. FASEB J. 2003; 17(8): 788-789, doi: 10.1096/fj.03-0093life, indexed in Pubmed: 12724335.

24. Dickstein K, Vardas PE, Auricchio A, et al. 2010 Focused Update of ESC Guidelines on device therapy in heart failure: an update of the 2008 ESC Guidelines for the diagnosis and treatment of acute and chronic heart failure and the 2007 ESC Guidelines for cardiac and resynchronization therapy. Developed with the special contribution of the Heart Failure Association and the European Heart Rhythm Association. Europace. 2010; 12(11): 1526-36.

25. Dickstein K, Cohen-Solal A, Filippatos G, et al. ESC guidelines for the diagnosis and treatment of acute and chronic heart failure 2008: the Task Force for the diagnosis and treatment of acute and chronic heart failure 2008 of the European Society of Cardiology. Developed in collaboration with the Heart Failure Association of the ESC (HFA) and endorsed by the European Society of Intensive Care Medicine (ESICM). Eur J Heart Fail. 2008; 10(10): 933-989, doi: 10.1016/j.ejheart.2008.08.005, indexed in Pubmed: 18826876.

26. Brignole M, Auricchio A, Baron-Esquivias G, et al. 2013 ESC Guidelines on cardiac pacing and cardiac resynchronization therapy: The Task Force on cardiac pacing and resynchronization therapy of the European Society of Cardiology (ESC). Developed in collaboration with the European Heart Rhythm Association (EHRA). Europace. 2013; 15(8): 1070-1118, doi: 10.1093/europace/eut206.

27. Mehta M, Jain A, Mehta A, et al. Cardiac arrhythmias following intravenous nicotine: experimental study in dogs. J Cardiovasc Pharmacol Ther. 2016; 2(4): 291-297, doi: 10.1177/1074248497 00200407 .

28. Goette A, Lendeckel U, Kuchenbecker A, et al. Cigarette smoking induces atrial fibrosis in humans via nicotine. Heart. 2007; 93(9): 1056-1063, doi: 10.1136/hrt.2005.087171, indexed in Pubmed: 17395670.
29. Shan H, Zhang Y, Lu Y, et al. Downregulation of miR-133 and miR-590 contributes to nicotine-induced atrial remodelling in canines. Cardiovasc Res. 2009; 83(3): 465-472, doi: 10.1093/cvr/ cvp130, indexed in Pubmed: 19398468.

30. Leone A. Biochemical markers of cardiovascular damage from tobacco smoke. Curr Pharm Des. 2005; 11(17): 2199-2208, indexed in Pubmed: 16026289.

31. Andre L, Boissière J, Reboul C, et al. Carbon monoxide pollution promotes cardiac remodeling and ventricular arrhythmia in healthy rats. Am J Respir Crit Care Med. 2010; 181(6): 587-595, doi: 10.1164/rccm.200905-0794OC, indexed in Pubmed: 20019346.

32. Plank B, Kutyifa V, Moss AJ, et al. Smoking is associated with an increased risk of first and recurrent ventricular tachyarrhythmias in ischemic and nonischemic patients with mild heart failure: a MADIT-CRT substudy. Heart Rhythm. 2014; 11(5): 822-827, doi: 10.1016/j.hrthm.2014.02.007, indexed in Pubmed: 24509214.

33. Goldenberg I, Moss AJ, McNitt S, et al. Cigarette smoking and the risk of supraventricular and ventricular tachyarrhythmias in high-risk cardiac patients with implantable cardioverter defibrillators. J Cardiovasc Electrophysiol. 2006; 17(9): 931-936, doi: 10.1111/j.1540-8167.2006.00526.x, indexed in Pubmed: 16759297.

34. Benowitz N. Pharmacology of Nicotine: Addiction, Smoking-Induced Disease, and Therapeutics. Annu Rev Pharmacol Toxicol. 2009; 49(1): 57-71, doi: 10.1146/annurev.pharmtox.48.113006.094742.

35. Benowitz NL. Clinical pharmacology of nicotine. Annu Rev Med. 1986; 37(1): 21-32, doi: 10.1146/annurev.me.37.020186.000321.

36. Fattinger K, Verotta D, Benowitz NL. Pharmacodynamics of acute tolerance to multiple nicotinic effects in humans. J Pharmacol Exp Ther. 1997; 281(3): 1238-1246, indexed in Pubmed: 9190859.

37. Falk JA, Kadiev S, Criner GJ, et al. Cardiac disease in chronic obstructive pulmonary disease. Proc Am Thorac Soc. 2008; 5(4): 543-548, doi: 10.1513/pats.200708-142ET, indexed in Pubmed: 18453369 . 